\title{
Measurement of the nonlinear refractive index in optical thin films
}

M. Steinecke, M. Jupé, K. Kiedrowski, D. Ristau

M. Steinecke, M. Jupé, K. Kiedrowski, D. Ristau, "Measurement of the nonlinear refractive index in optical thin films," Proc. SPIE 10447, LaserInduced Damage in Optical Materials 2017, 104472A (22 March 2018); doi: $10.1117 / 12.2281127$ 


\title{
Measurement of the nonlinear refractive Index in optical thin Films
}

\author{
M. Steinecke*a, M. Jupéa ${ }^{\mathrm{a}}$, K. Kiedrowski ${ }^{\mathrm{a}}$, D.Ristau ${ }^{\mathrm{a}, \mathrm{b}}$ \\ ${ }^{a}$ Laser Zentrum Hannover e.V., Laser Components Department, Hollerithallee 8, 30419 Hannover, \\ Germany \\ ${ }^{\mathrm{b}}$ Institut für Quantenoptik, QUEST, Leibniz Universität Hannover, Germany
}

\begin{abstract}
Based on the z-scan method, an interferometric set-up for measuring the optical Kerr-effect was engineered and optimized. Utilizing a Mach-Zehnder configuration, the wave front deformation caused by the Kerr induced selffocusing is monitored. Fitting this deformation to a theoretical approach basing on a beam propagation model, the nonlinear refractive index is obtained. The procedure can be applied to measure the nonlinear refractive index of both, the substrate material as well as the deposited dielectric layer on top of the substrate. The nonlinear refractive index of a layer specially deposited for this purpose as well as for several substrate materials was measured and the results presented.
\end{abstract}

Keywords: Kerr-effect, self-focusing, thin films, nonlinear optics

\section{INTRODUCTION}

In the past years, the demands on optical components have increased continuously. This increase can be divided into two different directions. On one hand, ultra-short-pulse applications demand the management of broad spectra, combined with a management of the spectral phase for GDD-control [1]. This requires a precise control of the coating process and extensive knowledge of layer material properties. On the other hand, the available laser power keeps increasing and so the power handling capabilities of optics are moving more and more into the focus of attention [2].

These two developments necessitate the consideration of nonlinear optical effects in the coating stack, as they gain in importance with higher incident powers and can influence the function of a coating stack in different ways. Of major significance are nonlinear effects of the third order, as they, contrary to effects of second order, occur in amorphous media, which typically constitute optical layer stacks. An example is the optical Kerr-effect, which changes the refractive index depending on the intensity of the incident radiation and a material constant, the so called nonlinear refractive index. This effect can either change the spectral properties of an optical component [3] or contribute to the destruction of the component via the so called self-focusing.

To compensate or even exploit the nonlinear effects occurring in thin film layer stacks, a precise knowledge of the corresponding material constants is essential. As the linear optical properties of materials as thin films are known to differ from the linear optical properties of the corresponding bulk materials [4], it is reasonable to assume this also to be true for the nonlinear properties of optical layers, so that using the values known from bulk materials could cause errors when dealing with the effects in thin film stacks. To measure the nonlinear properties of optical layers, either a high nonlinear coefficient or a sufficient propagation length in the investigated material is necessary, with typical materials for optical thin films requiring propagation lengths of over $50 \mu \mathrm{m}$. To create optical films with thicknesses in this order of magnitude and the desired optical quality, the coating process and treatment of the samples has to be optimized thoroughly. Most prominently, the layer stress as well as the absorption of the layer material has to be minimized to reach the desired mechanical stability and optical transmission. To achieve this, the layers were deposited applying an Ion-Beam-Sputtering process in combination with in-situ substrate heating as well as ex-situ annealing.

*m.steinecke@1zh.de; phone +49 5112788297

Laser-Induced Damage in Optical Materials 2017, edited by Gregory J. Exarhos, Vitaly E. Gruzdev,

Joseph A. Menapace, Detlev Ristau, MJ Soileau, Proc. of SPIE Vol. 10447, 104472A

(C) 2017 SPIE · CCC code: $0277-786 X / 17 / \$ 18 \cdot$ doi: $10.1117 / 12.2281127$ 
In addition to the necessary layer qualities, a precise measurement procedure is necessary to determine the nonlinear refractive index. Therefore, a new approach for measuring the nonlinear refractive index of single layer thin films as well as bulk materials was developed and tested. The approach is based on the classical z-scan technique [5] combined with a Mach-Zehnder-interferometer [6], which allows the monitoring of the wave front deformation while varying the intensity of the radiation incident on the sample. A simulation procedure allows fitting of the measured data, and consequently the nonlinear refractive index of the sample can be determined. In this work, the measurement technique and the simulation process are explained, and measurement results for optical bulk materials as well as single layers are presented and discussed.

\section{THEORY AND SIMULATION PROCEDURE FOR THE KERR-EFFECT IN OPTICAL MEDIA}

\subsection{Nonlinear refractive Index and Self-Focusing}

The nonlinear polarization of third order causes many different effects in optical media [7]. One of these effects is the change of refractive index $\mathrm{n}$ proportional to the applied radiation intensity $I$ and the material dependent nonlinear refractive index $\mathrm{n}_{2}$ as presented in the following equation:

$$
n=n_{0}+n_{2} * I \text {. }
$$

When radiation with a radially symmetric but not uniform intensity distribution in beam cross section (e.g. a Gaussian $\mathrm{TEM}_{00}$-Mode, see also [8]), interacts with a medium which exhibits this third order effect, a phase shift corresponding to the intensity distribution occurs. For a Gaussian beam, the intensity is at its maximum in the center of the beam, and decreases with greater distances to the center. This produces a laterally varying refractive index with (for positive $\mathrm{n}_{2}$ ) a maximum in the center of the beam and the undisturbed refractive index of the material $\mathrm{n}_{0}$ at the beam edges. This refractive index profile causes a phase shift similar to that of a lens i.e. the beam gets focused by the self-induced refractive index profile. This so called self-focusing is illustrated in figure (1). For media, which are thin compared to the Rayleigh-length of the applied beam, the self-focusing effect can be approximated by viewing the medium as a thin lens with the focal length $f_{\text {Kerr }}$, with

$$
f_{\text {Kerr }}=\frac{a \pi w^{4}}{4 n_{2} d P}
$$

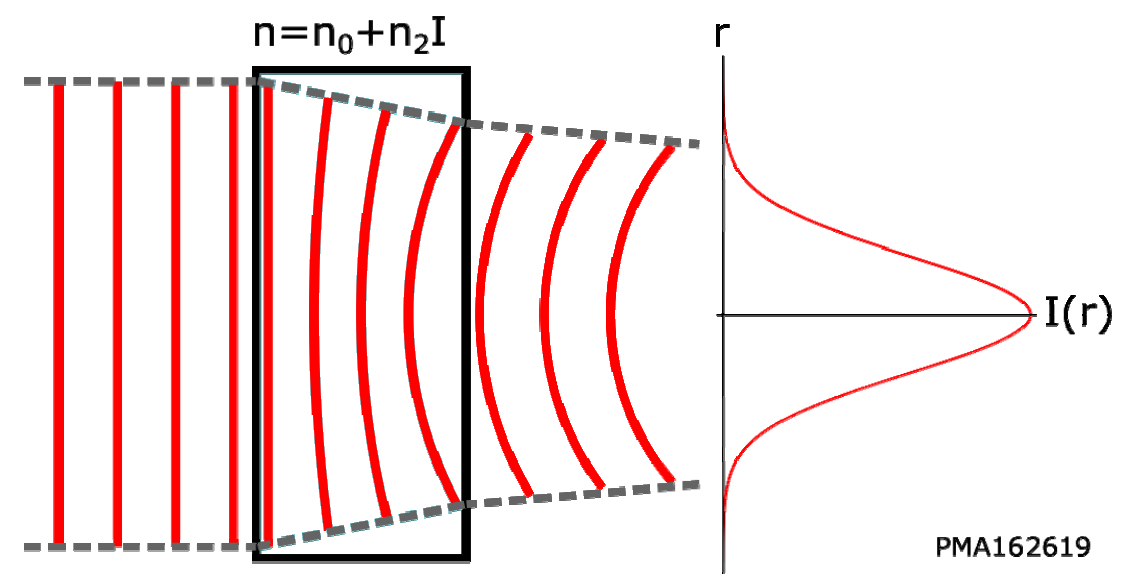

Figure 1: Because of the inhomogeneous intensity distribution, the refractive index in the center of the medium is increased significantly than on the outside, at the beam's edges. This causes a self-focusing effect of strong laser beams in optical media. 
Hereby, $P$ is the power of the incident radiation, $d$ the thickness of the irradiated area along the beam path, $w$ the beam radius at the sample position and $a$ is a correction factor, which accounts for the difference between the Gaussian beam shape and a parabolic beam profile [9].

\subsection{Simulation of Self-Focusing in optical Media}

A well-established method for measuring the nonlinear refractive index of optical bulk materials is the so-called z-scan [5]. The method is based on the change of the beam diameter caused by the self-focusing in the sample while moving the sample along the path of a focused beam. This change of beam diameter is converted into a measurable power variation by measuring the radiation transmitted through a small pinhole. The setup is illustrated in figure (2). The sample movement along the path of the focused beam continuously changes the intensity of the radiation, and therefore the detected power also changes. Figure (3) shows a characteristic curve for a z-scan measurement of the Kerr-effect. The zscan relies on a modeling of the phase front deformation for evaluation of the measured data, with the assumption that the thickness of the sample is small enough, so that changes of the beams diameter in the sample can be neglected. For a more general approach, the occurring influence on the beam propagation is simulated with the optical matrix formulism [10]. For this, two matrices are needed:

$$
\begin{aligned}
& \mathrm{M}_{\mathrm{P}}(\mathrm{d}, \mathrm{n})=\left(\begin{array}{cc}
1 & \mathrm{~d} / \mathrm{n} \\
0 & 1
\end{array}\right) \text {, and } \\
& M_{\mathrm{L}}(f)=\left(\begin{array}{cc}
1 & 0 \\
-1 / f & 1
\end{array}\right) .
\end{aligned}
$$

The matrix $M_{\mathrm{P}}(d, n)$ represents a propagation of a distance $d$ through a medium with refractive index $n$, and the matrix $M_{\mathrm{L}}(f)$, which represents the focusing of a thin lens with focal length $f$. The beam properties can then be calculated by viewing the complex beam parameter $q(z)$, with

$$
\begin{aligned}
& \frac{1}{q(z)}=\frac{1}{R(z)}-\frac{i \lambda}{\pi n w(z)^{2}}=\left(z+i z_{\mathrm{R}}\right)^{-1}, \text { and } \\
& z_{\mathrm{R}}=\frac{\pi w_{0}^{2}}{\lambda} .
\end{aligned}
$$

Hereby, $R(z)$ is the radius of curvature (ROC) of the beam's wave front, $i$ the imaginary unit, $\lambda$ the radiation's wavelength, $n$ the refractive index of the medium, $w(z)$ the beam radius and $z_{\mathrm{R}}$ is the Rayleigh range of the beam with $w_{0}$ as beam diameter in the beam's focus.

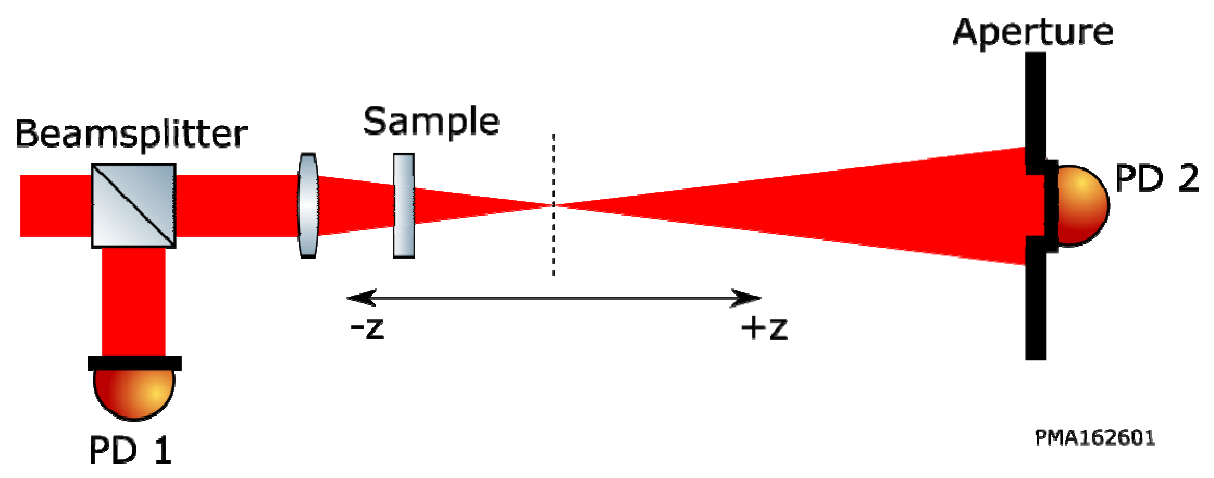

Figure 2: The z-scan set-up: The laser beam is focused and the sample is moved along the beam to vary the incident intensity, which in turn varies the power transmitted through the aperture. The laser power is monitored with photo detector (PD 1), and the transmitted power is measured with PD 2. 


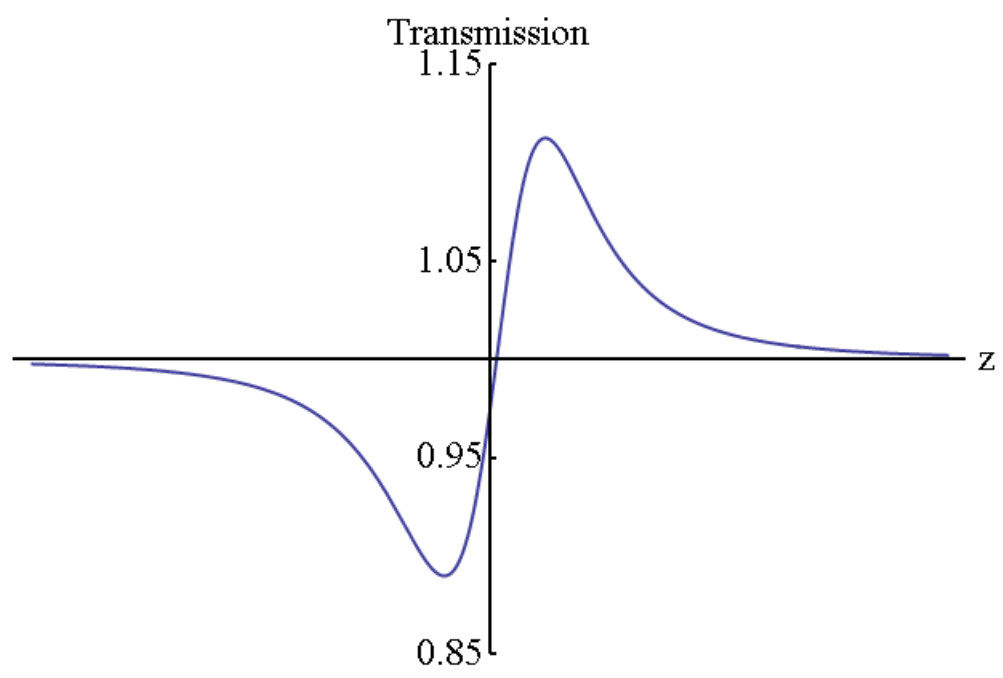

Figure 3: A typical signal created by a z-scan for a sample with positive $\mathrm{n}_{2}$. The changing focal length of the Kerr-lens in the sample caused by the varying intensity during the sample movement causes different beam diameters on the aperture and therefore the transmitted power changes with the sample position. When the sample is in front of the beam's focus, the intensity is lowered, and after the beam's focus, the intensity is increased because of the smaller beam diameter. The transmitted power is normalized to the undisturbed level.

With a known initial beam parameter $q_{\mathrm{in}}$, and an optical system characterized by an optical matrix $M$ with the components

$$
M=\left(\begin{array}{ll}
A & B \\
C & D
\end{array}\right),
$$

one can calculate the resulting optical beam parameter $q_{\text {out }}$ via the following relation [12]:

$$
\frac{1}{q_{\mathrm{out}}}=\frac{C+D / q_{\text {in }}}{A+B / q_{\text {in }}}
$$

This generalized calculation method allows the simulation of the Kerr-effect for differing setups. Figure (4) shows a typical z-scan profile for a thin sample, one time simulated with the classical wave-optical procedure (compare [12]) and one time with the matrix approach. The different methods of simulation methods yield almost identical results.

Often, the intensities necessary to achieve a significant impact of the self-focusing require a strong focusing of the applied radiation. This leads to short Rayleigh-ranges for the focused beams and therefore limits the thickness of samples, for which the approximation as a thin lens is valid. The simulations performed for thicker samples would consequently not match the measurements. To avoid that inaccuracy, the matrix formulism can be applied in combination with the so called distributed lens approximation [13]. Samples with thicknesses in the range of the Rayleigh-length are approximated as a sequence of Kerr-lenses $L_{i}$ followed by a short propagation $\Delta L$ through a Kerrinactive medium (see figure (5) for illustration). The propagation length following each Kerr lens is chosen short enough, so that the thin lens approximation is valid again, and the sequential calculation accounts for beam changes through selffocusing while the beam propagates inside the medium. Therefore, the self-focusing inside of thick media can be considered with an iterative procedure, in which the beam parameter is calculated after each sequence of $L_{i}$ and $\Delta L$, and the resulting beam parameters are then used to calculate the focal length of the next Kerr-lens. This process is repeated, until the necessary number of sequences is reached and the beam exits the Kerr-active medium. 


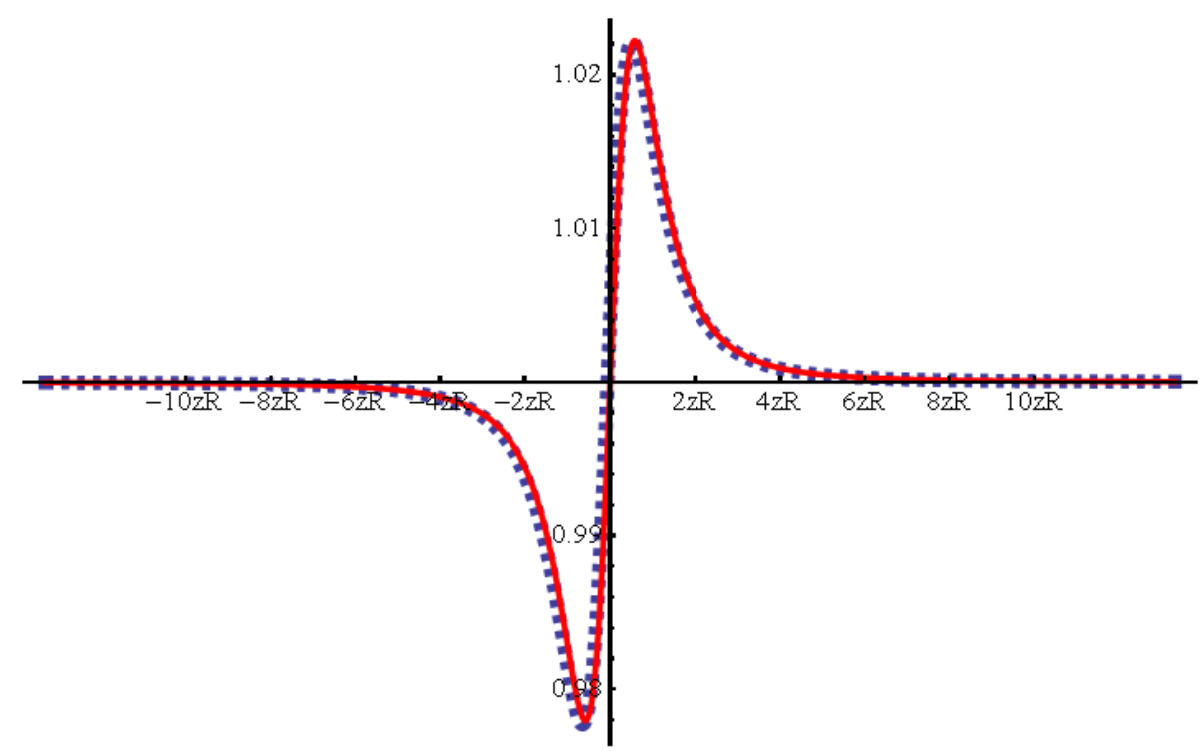

Figure 4: Two z-scan curves, simulated with the wave optical approach (solid, red) and the optical matrix formalism (dashed, blue). On the ordinate, the simulated, relative power variation on the detector, and on the abscissa the sample position in Rayleigh-lengths $z_{\mathrm{R}}$ is plotted. As the graph shows, two methods yield almost identical results.

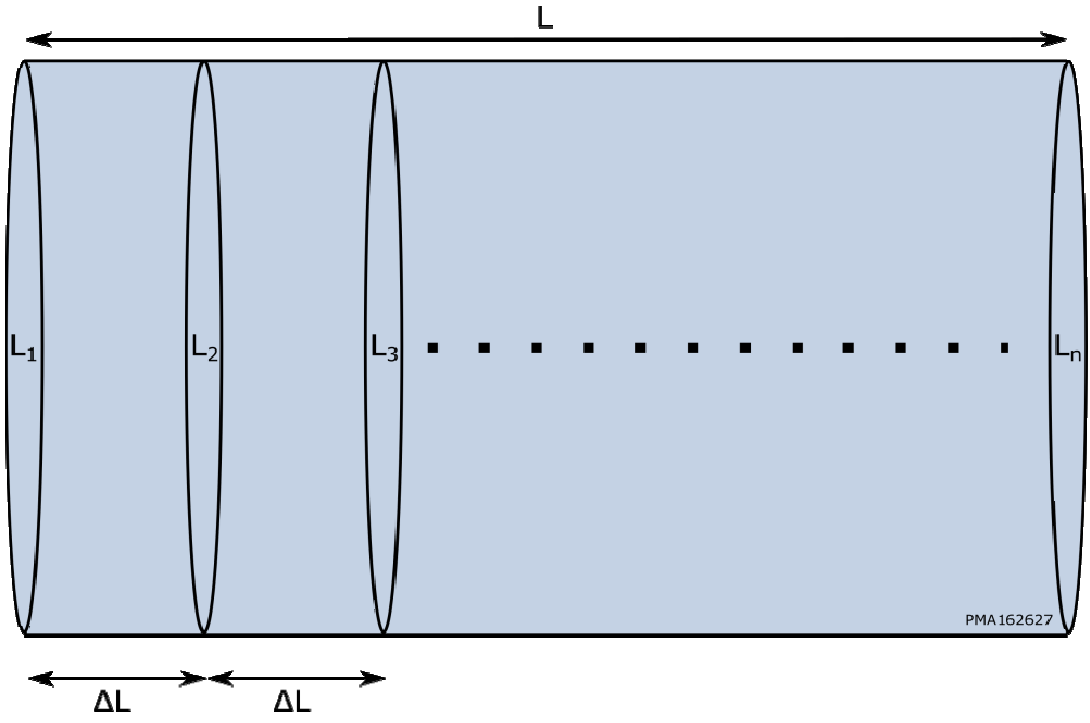

Figure 5: An illustration of the thin lens approximation. Substrates which are not significantly thinner than the Rayleigh length of the focused beam, can be calculated as sequence of Kerr-lenses $L_{i}$ and propagation lengths $\Delta L$. Each section of lens and propagation length itself is the thin enough to justify the thin-lens-approximation, and the effect of thick substrates can be calculated by calculation the effect of the complete sequence. 


\section{SIMULATIONS \& INTERFEROMETRIC SET-UP FOR MEASURING THE NONLINEAR REFRACTIVE INDEX}

As mentioned in section 2, the z-scan is an established method for measuring the nonlinear refractive index of optical bulk materials. The z-scan utilizes the change of beam diameter $w(z)$ caused by the self-focusing occurring in the sample. As can be seen in equation (2.4) the beam diameter is part of the imaginary part of the complex beam parameter. As an alternative the real part of the complex beam parameter, the ROC of the transmitted beam, can be measured to calculate the nonlinear refractive index. This parameter has a much bigger dynamic range, as the ROC can vary from values in the range of centimeter to meter at the maximum curvature of a Gaussian beam to infinity at focus locations. This greater range in principle allows for a more sensitive measurement of the beam deformation than the change in beam diameter, which only causes an intensity variation in the order of several percent. Another advantage results from the decoupling of the measured beam parameter from the power transmitted through the investigated material. In opposite to the classical z-scan approach, where a precise measurement of the power transmitted through the aperture is essential, the measurement of the ROC does, in principle, not depend on the transmitted power. This strongly reduces the potential sensitivity for absorption effects, such as the nonlinear absorption occurring at high power levels.

\subsection{Simulation of the Measurement Procedure}

The simulation process described in section (2) can be used to investigate the potential advantages of the transmitted beams ROC as monitored parameter. An exemplary setup for measuring the wave front curvature is presented in figure (6). The sample is moved through a focused beam in an optical telescope, and a wave front detector is monitoring the ROC of the wave front. The wave front detector could be realized in different forms, for example using a HartmanShack-Sensor [14] or, as presented in section (3.2), by an interferometric set-up. By modeling the different steps the beam takes (propagation from the lens to the sample, the Kerr-effect in the sample as simulated by the distributed lens approximation, propagation from the sample to the second lens, the second lens itself and the propagation to the detector), the ROC can be calculated for each sample position. Figure (7) shows exemplary simulations for a sample with a thickness of $1 \mathrm{~mm}$, and a sample with the same nonlinear refractive index but with thickness of $6 \mathrm{~mm}$. The signal caused by the two samples has a significantly different shape: with the thinner sample producing one, and the thicker sample two distinct peaks.

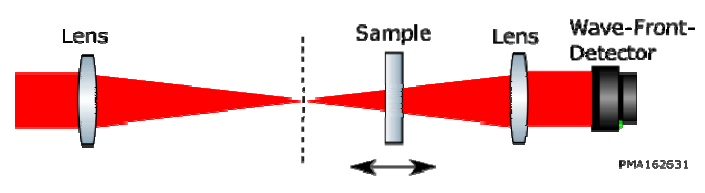

Figure 6: The concept for a wave front-based measurement. The sample is placed in a telescope which provides the focused beam. By moving the sample along the beam, the self-focusing changes the wave front on the detector.

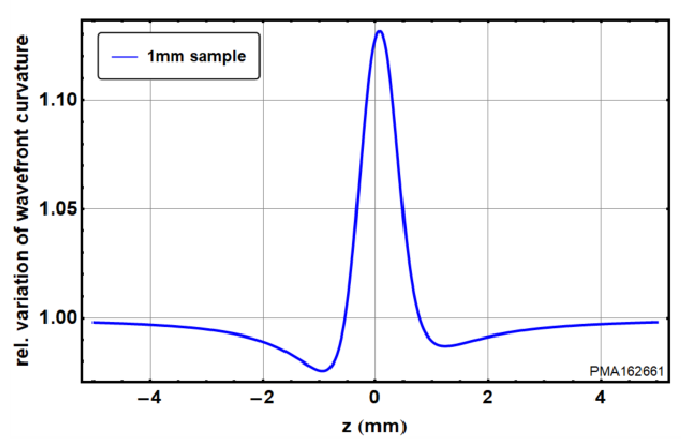

(a)

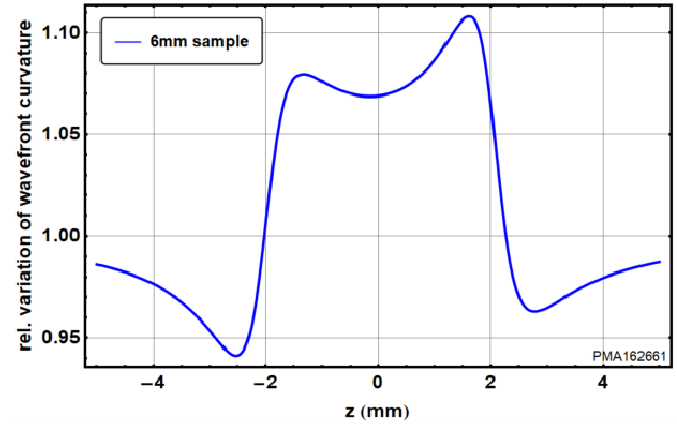

(b)

Figure 7: Exemplary simulations for a thin $(1 \mathrm{~mm})$ and a thick $(6 \mathrm{~mm})$ optical bulk substrate. The variation of the wave front curvature shows a clearly different profile for the different thicknesses, with the thin substrate producing one peak, and the thick one two. 


\subsection{Interferometric Setup and Laser System}

For the measurement of the wave front curvature of an optical beam transmitted through a Kerr-active sample, an interferometric setup (presented in figure (8)) was constructed. The setup is based on a Mach-Zehnder-interferometer, where one of the arms is used for a reference beam while the sample is placed in a focused beam in the other arm. Before entering the interferometer itself, the beam is collimated to initially create a very plane wave front. The reference beam then proceeds unchanged to a camera, which is used to record the resulting interference pattern. The beam in the samplearm is focused for greater intensity, and, after passing the sample, the beam is collimated again to allow a superposition.

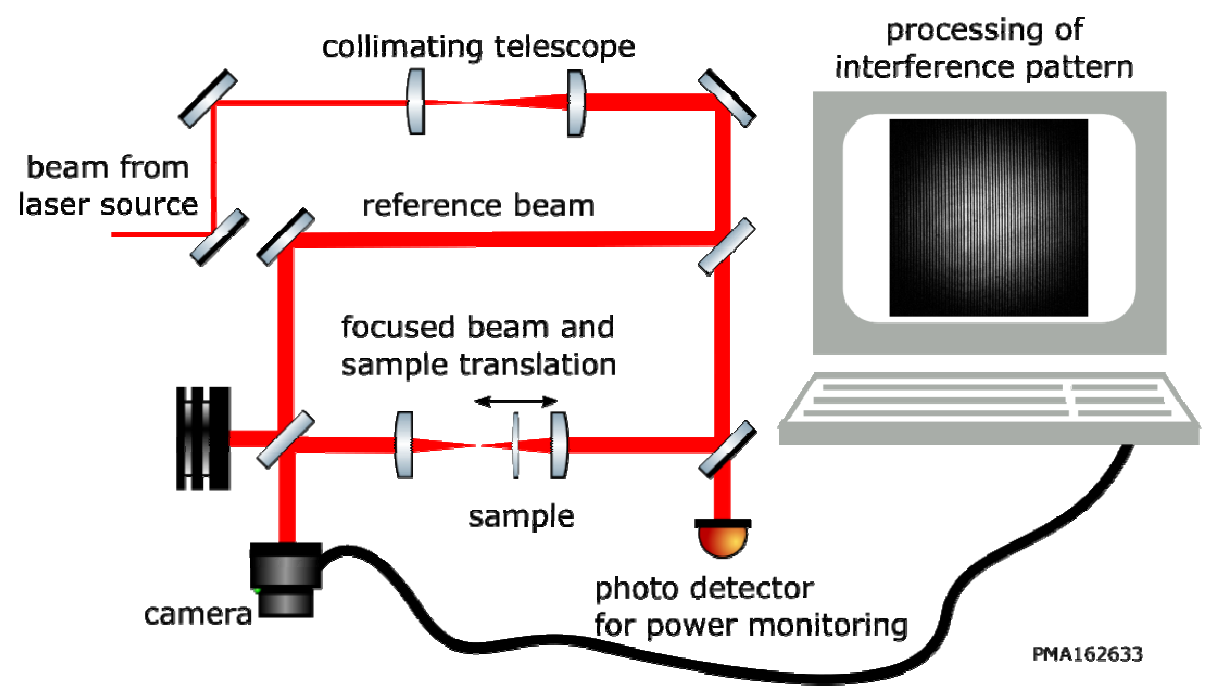

Figure 8: The interferometric setup: The laser beam is collimated and split into the two interferometer arms. The sample is moved along a focused beam inside the telescope in one arm, while the other arm provides a reference wave front. The interference pattern is then recorded by a digital camera.

The experiments were conducted with a LUMERA Rapid $\mathrm{Nd}: \mathrm{YVO}_{4}$-Laser emitting at $1064 \mathrm{~nm}$ with a repetition rate of $10 \mathrm{kHz}$, a pulse length of $12 \mathrm{ps}$ and a pulse energy of up to $100 \mu \mathrm{J}$. The focus diameter in the telescope was $28 \mu \mathrm{m}$ and the $\mathrm{M}^{2}$-factor of the beam was measured as 1.04 .

\subsection{Retrieval of Wave Front Curvature}

For the retrieval of the wave front curvature, a Fourier-transform phase demodulation method with a spatial linear carrier is used [15]. To achieve the linear carrier, the two beams are superimposed under a small angle, which changes the interference pattern (see figure (9a)) to a sequence of stripes. The recorded interference pattern is then transformed by a 2D-Fast-Fourier-Transformation (FFT) to obtain the spatial frequency spectrum. A typical spectrum is presented in figure (9b). The spectrum consists of a central maximum in intensity and two symmetrical maxima at the location determined by the spatial carrier. These maxima contain the information about the wave front curvature.

To obtain the information, a filter is applied which isolates one of the maxima. The selected maximum is then shifted to the center of the spectrum and an inverse 2D-FFT is utilized for obtaining the desired spatial image. The complex phase of the resulting image represents the phase difference between the two beams, and as the reference beam possesses a practically plane wave front, this yields the phase distribution of the sample beam. A typical phase distribution obtained with this calculation method is presented in figure (10). To calculate the ROC of the wave front from the phase distribution, the $2 \pi$-phase jumps need to be compensated. As this is complicated and not always possible in $2 \mathrm{D}$, it was performed by reading the intensity values from the phase distribution images along 1D-lines. A schematic of the read lines' distribution is also shown in figure (10), in the presented measurements, 100 lines were used for all evaluations. The lines are arrayed in a star-like shape with the center of each line in the center of the phase distribution. The $2 \pi$-phase jumps for each line can then be compensated easily. A circular fit yields the ROC for each line and the average ROC of all lines is the resulting value for the wave front of the transmitted beam. The recording of the interference images as well as the retrieval of the wave front's ROC were programmed for this purpose with the software LABView from National Instruments. 


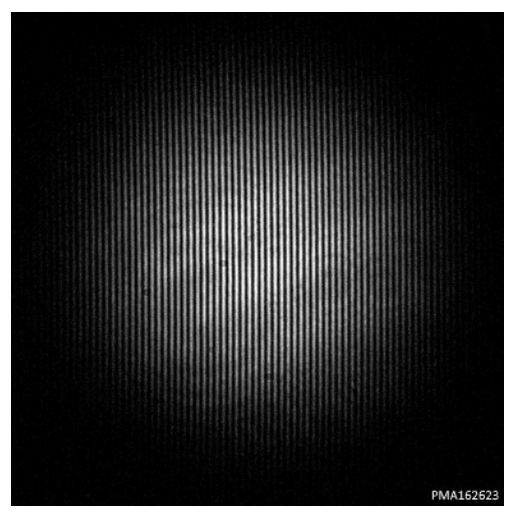

(a)

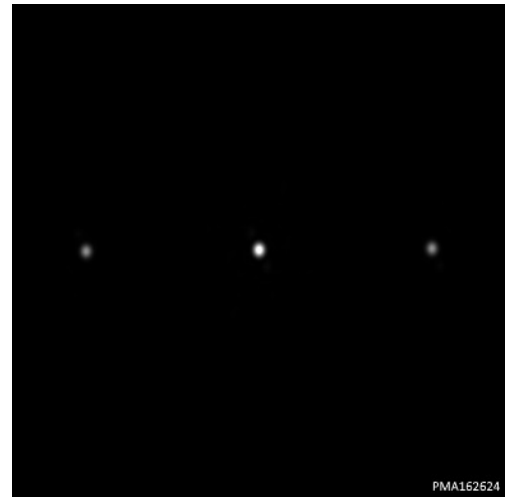

(b)

Figure 9: The interference pattern (a) created by superimposing the beam transmitted through the sample and the reference beam. A 2-dimensional FFT is applied to obtain the spatial spectrum (b). The spectrum clearly shows a central maximum and two, symmetric maxima, caused by the interference pattern.

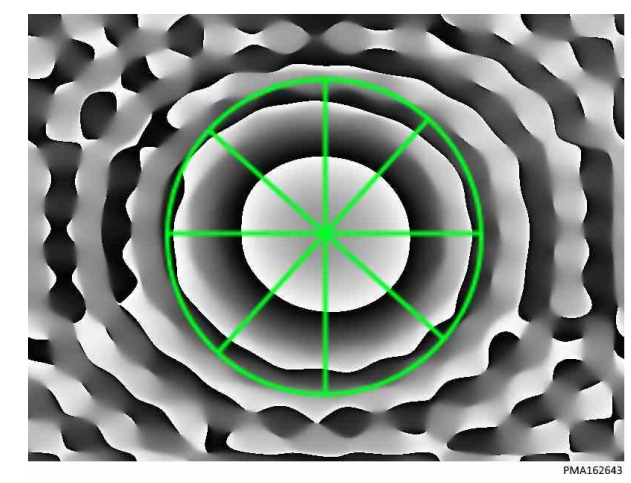

Figure 10. The phase distribution retrieved from the interference pattern. The green pattern represents schematically the lines used for evaluation of the ROC.

\section{MEASUREMENTS OF THE NONLINEAR REFRACTIVE INDEX FOR BULK MATERIALS}

To quantify the developed method for measuring the nonlinear refractive index, several types of optical bulk material were measured. For the measurements to be accurate, the correction factor $a$ from equation (2.1) needs to be determined. This can be done by measuring a material with a well-known nonlinear refractive index, such as sapphire $[16,17,18]$. The measurement of a sapphire sample with a thickness of $1 \mathrm{~mm}$ and the corresponding simulation is shown in figure (11). With the set-up calibrated in this fashion, more materials with different thicknesses were investigated and simulations were used to determine the nonlinear refractive index of the material, as this is the only free parameter remaining. The measurements and simulations are presented in figure (12) and the resulting values for the nonlinear refractive index are summarized in table (1). The table also shows corresponding values from the literature for comparison. The measurements show overall a good agreement with the simulations, and the calculated nonlinear refractive indices match with published data. 


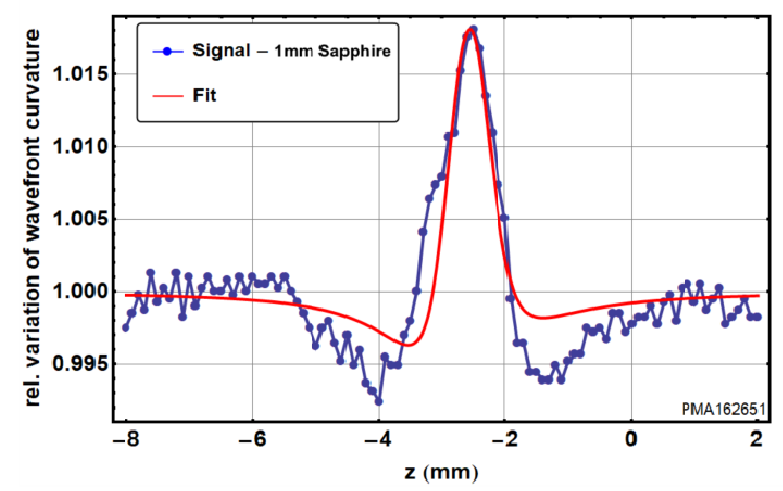

Figure 11. The measurement results for a sapphire substrate. As the nonlinear refractive index for sapphire glass is relatively well known $[15,16,17]$, it can be used to calibrate the measurement by determining the correction factor $a$ from equation (2.1).

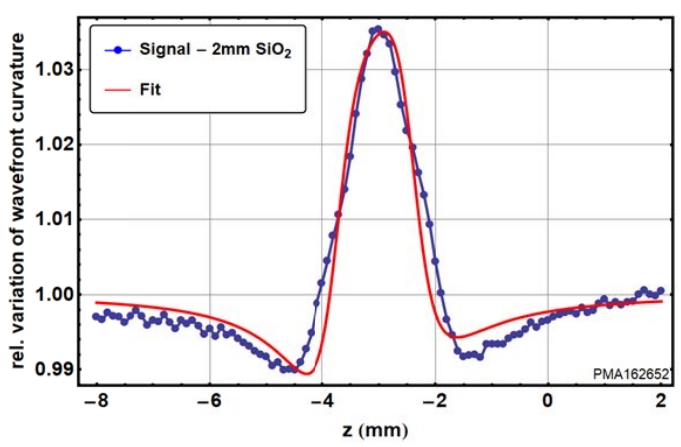

(a)

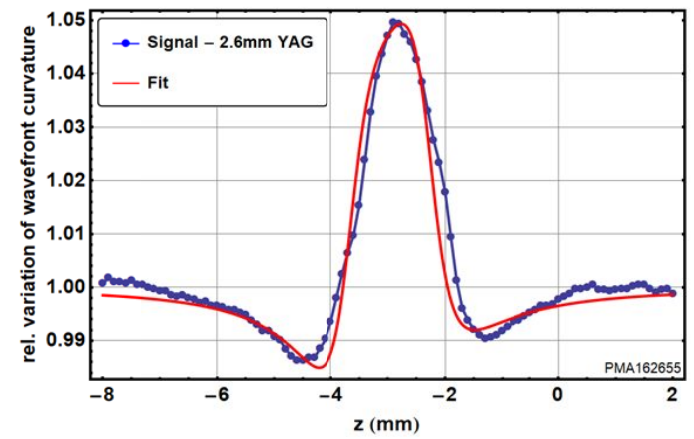

(c)

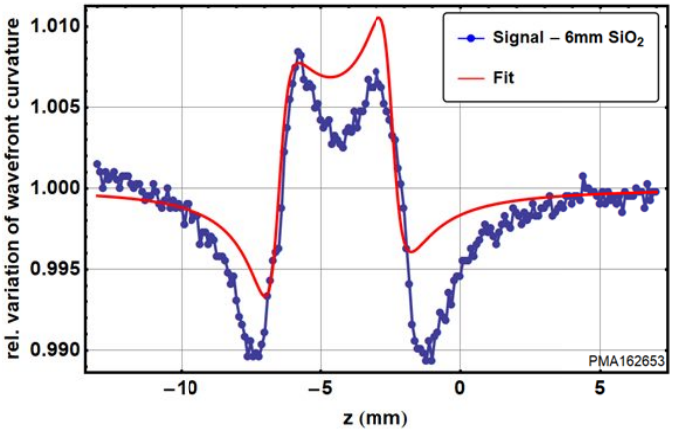

(b)

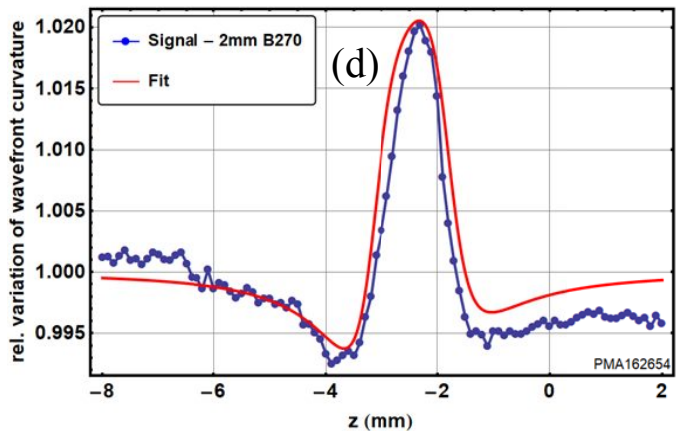

(d)

Figure 12: Measurements of optical bulk materials with the wave front based set-up. In each graph, the relative wave front curvature variation is plotted against the sample position variation. The blue connected dots represent the measured data, and the red line corresponds to the calculated fit. 
Table 1: Measurement results for the nonlinear refractive index of optical bulk materials and literature values for comparison. Overall, the measured values are in reasonable agreement with the literature.

\begin{tabular}{|c|c|c|c|c|}
\hline Material & Thickness $(\mathrm{mm})$ & $\mathrm{n}_{0} @ 1064 \mathrm{~nm}$ & $\mathrm{n}_{2} \cdot\left(10^{-20} \mathrm{~m}^{2} / \mathrm{W}\right)$ & $\mathrm{n}_{2}$ lit $\left(10^{-20} \mathrm{~m}^{2} / \mathrm{W}\right)$ \\
\hline $\mathrm{SiO}_{2}$ & 2 & 1.4496 & 4.3 & $1.9-3[19]$ \\
\hline $\mathrm{SiO}_{2}$ & 6 & 1.4496 & 3.4 & $1.9-3[19]$ \\
\hline YAG(@1300) & 2.6 & 1.8147 & 7.1 & $7.7[20]$ \\
\hline B270 Glas(@620) & 2 & 1.5186 & 3.3 & $3.4[21]$ \\
\hline
\end{tabular}

\section{MANUFACTURING AND MEASUREMENT OF OPTICAL THIN FILMS}

To create optical thin films with the required thickness of over $100 \mu \mathrm{m}$ and high quality for the measurement of the nonlinear refractive index, two main factors have to be kept in mind and optimized: The layer stress and the absorption. If the layer stress is too high, the optical film will, when a certain thickness is reached, either break the substrate or delaminate. When the absorption in the optical film is not low enough, the film will, because of its very high thickness, absorb a significant amount of the laser radiation applied for measuring purposes.

To create the layers, an Ion Beam Sputtering (IBS) deposition with the special co-sputtering technique was applied [22]. While IBS processes are used to create high quality, the typical deposition rate of these processes is rather low. However, for the multi-micrometer coatings presented here, the deposition rate could be increased to approximately one $\mathrm{nms}^{-1}$ for a small area by optimizing the substrate position. The application of the co-sputter technique allows manufacturing mixtures of two coating materials by using a target translation mount to laterally move the target material relative to the ion beam. The ratio of the areas the ion beam hits on the two target materials then determines the ratio of the sputtered materials. The atoms sputtered from the target materials are then oxidized by inserted oxygen gas and create a ternary oxidic layer on the substrates. In this work, a commercial Veeco RF high power ion source was used, and the sputtering is performed with argon gas.

As an addition to this set-up, a heating element is implemented into the coating process. Usually IBS is a so called "cold" coating process, but, as the substrate temperature can have a significant influence on minimizing layer stress and absorption, a powerful heater was used for the manufacturing of layers in this work.

Several materials were investigated for their layer properties. As an example, the results for stress and absorption for a mixture of silicon oxide and aluminum oxide are presented in figure (13). The layers were deposited with substrate heating during the coating process as well as with post process annealing. Post process annealing was able to reduce the layer stress significantly for samples manufactured without heating, while the heating during the process reduces the initial stress strongly. The absorption values for unheated samples also improve with post-process annealing, but heated samples possess lower absorption values, which in turn do not change with post process annealing.

Overall, heating during the coating process seems to reduce the layer stress in the process, which allows for thicker coatings without interruptions for annealing, while also reducing the absorption in the manufactured layers. This knowledge was applied to manufacture layers of different material mixtures for investigation of the nonlinear refractive index of the coating material.

\subsection{Measurement of thin films}

To measure the nonlinear refractive index of optical thin films, different layers with thicknesses of approximately $100 \mu \mathrm{m}$ were deposited on quartz substrates of different thicknesses. While investigating the properties of the so produced samples, it became clear that thick $(6 \mathrm{~mm})$ substrates are more practical for measuring the layer properties. As can be seen in figure (7) and (12), thick substrates produce a signal with two distinguished peaks, which are caused by the surfaces of the sample. When an optical layer is deposited on one of these surfaces, the corresponding peak will change, while the other peak almost perfectly retains its shape. 


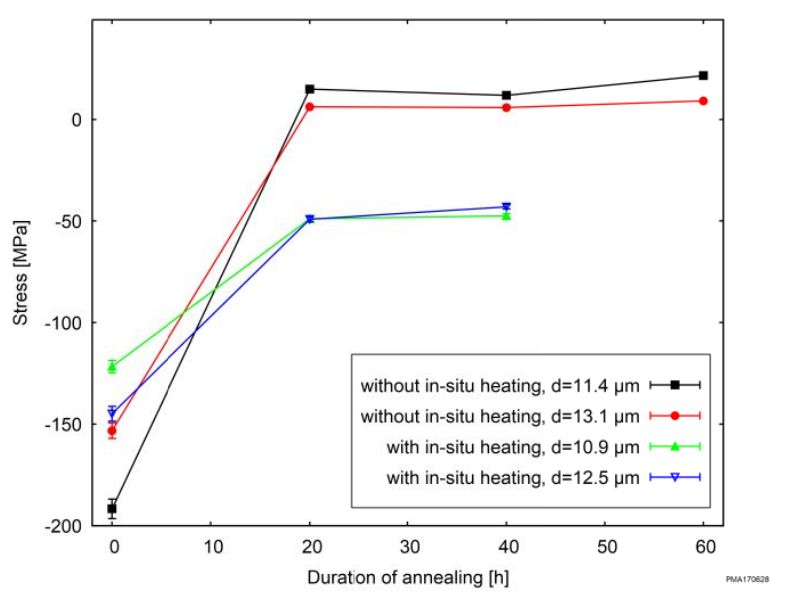

(a)

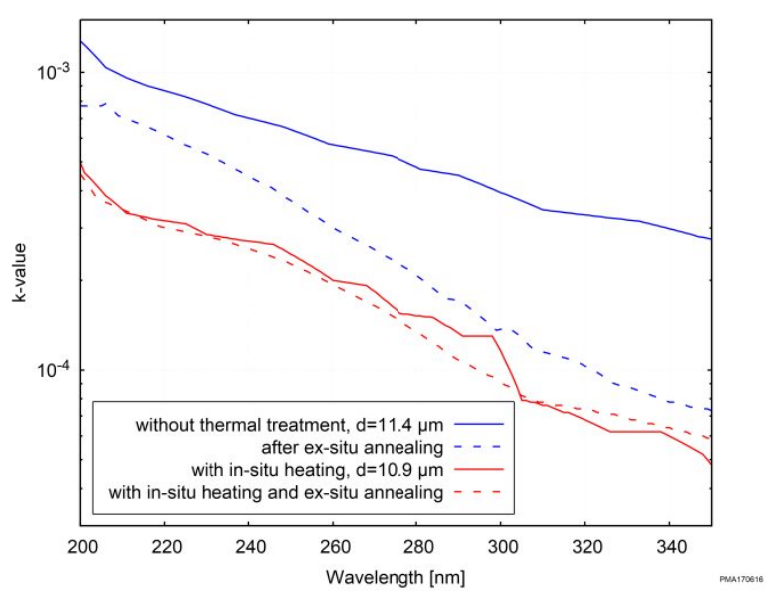

(b)

Figure 13: The influence of thermal treatment during and after the coating on layer stress and absorption. In (a) the layer stress for four samples with and without in-situ heating is compared. In-situ heating reduces the initial stress, yet the remaining stress after ex-situ annealing is higher. In (b) the relative absorption is presented. Here the sample with in-situ heating shows a much lower overall absorption, and ex-situ annealing has no significant influence. When no in-situ heating is applied, annealing is able to lower the absorption, yet not to the level of the heated sample.

An example for this procedure is presented in figure (14). The sample consists of a $6 \mathrm{~mm}$ quartz substrate coated on one side with an $85 \mu \mathrm{m}$ layer of niobium oxide (91\%) mixed with aluminum oxide (9\%). When compared with figure (12), the difference becomes obvious. A $6 \mathrm{~mm}$ substrate without coating shows two peaks of roughly the same height. The coating deposited on one of the surfaces will increase the height of the corresponding peak. By first fitting the left peak with the nonlinear refractive index of the substrate and then fitting the right peak with the nonlinear refractive index of the coating material, both parameters can be determined from a single measurement. The value measured for this coating material is $21.6 * 10^{-16} \mathrm{~cm}^{2} \mathrm{~W}^{-1}$, which is slightly higher than the value known from literature for bulk niobium oxide, which is $7.3 * 10^{-16} \mathrm{~cm}^{2} \mathrm{~W}^{-1}$ [23]. The difference may either result from the mixture of two materials or from the coating process itself.

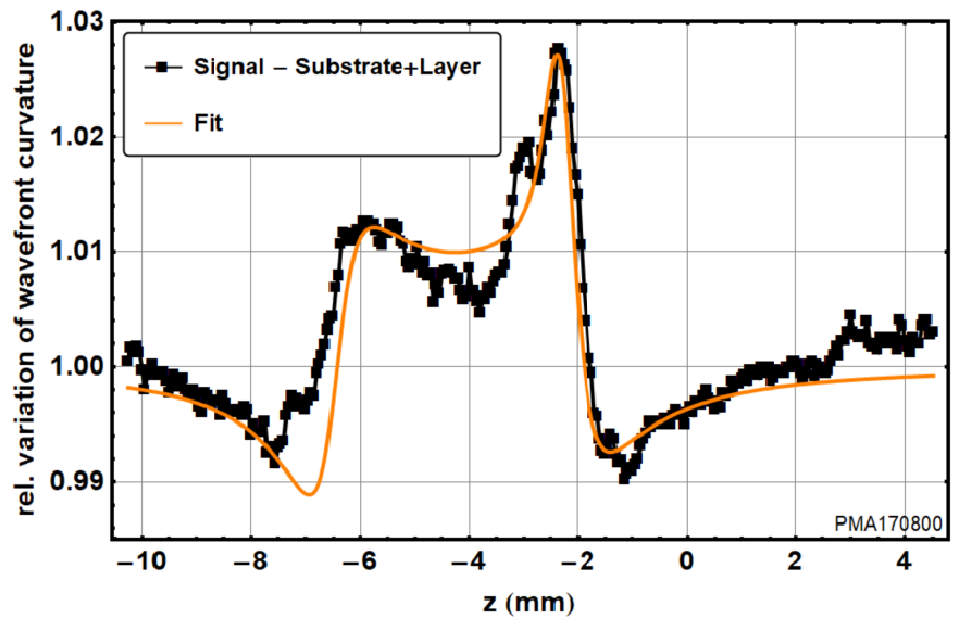

Figure 14: The measurement results with the fit for $\mathrm{n}_{2}$ determination for a coated sample. In comparison with figure (12b), one of the two peaks is increased in height by the layer deposited on the substrate. The sample presented in this figure is a $6 \mathrm{~mm} \mathrm{SiO}_{2}$-Substrate with a $85 \mu \mathrm{m}$ layer of niobium oxide $(91 \%)$ mixed with aluminum oxide $(9 \%)$. 


\section{CONCLUSION}

In this work, a novel measurement method for the nonlinear refractive index is presented and evaluated. The measurement is based on the established z-scan approach, but uses an interferometric procedure to monitor the wave front deformation instead of the classical power monitoring. This new approach could provide a higher sensitivity and is less influenced by nonlinear absorption caused by excited electrons in the material. Consequently, the measurement approach expands the available power range for investigations both, in the low power range resulting from a higher sensitivity and for intensities close to the damage thresholds because of the tolerance to nonlinear absorption effects. For evaluation of the measured wave front curvatures, the optical matrix formulism is applied to model the Kerr-lens selffocusing occurring in the measured sample. A special approach is used for the consideration of thick samples. The measured results show, that the method is suitable for the measurement of the nonlinear refractive index of optical bulk material as well as of deposited thin films. This will enable a more detailed knowledge of the nonlinear properties of coating materials and, in this way, allow a compensation or even exploitation of nonlinear effects in dielectric layer stacks.

\section{ACKNOWLEDGEMENTS}

The authors thank the Deutsche Forschungsgemeinschaft (DFG) for financial support in the cluster of excellence 201 QUEST and the Chi3-project (contract no. RI 645/2-2). Furthermore the authors thank the German Federal Ministry of Education and Research (BMBF) for the financial support of the research projects "Kerr band switch" (contract no. 13N13967) and the project "THG-layers" (contract no. 13N14063).

\section{REFERENCES}

[1] Pervak, V., Razskazovskaya, O., Angelov, I., Vodopyanov, K. and Trubetskov, M., "Dispersive mirror technology for ultrafast lasers in the range 220-4500 nm. Advanced Optical Technologies." Adv. Opt. Techn. 0, 1-9. (2013)

[2] Ristau, D. [Laser Induced Damage in Optical Materials], CRC Press, Boca Raton (2014)

[3] Fedulova, E., Trubetskov, M., Amotchkina T., Fritsch K., Baum, P., Pronin, O., and Pervak, V. "Kerr effect in multilayer dielectric coatings" Opt. Express 24(19), 21802-21817 (2016)

[4] Rao, M. C. and Shekhawat, M. S. "A brief Survey on Basic Properties of thin Films for Device Application" Int. J. Mod. Phys. Conf. Ser. 22, 576 (2013).

[5] Sheik-Bahae, M., Said, A. A. and Stryland, E. W. V., "High-sensitivity, single-beam n2-measurements." Opt. Lett. 14(17), 955-957 (1989)

[6] Zehnder, L. "Ein neuer Interferenzrefraktor" Zeitschrift für Instrumentenkunde 11, 275-285 (1891)

[7] Boyd, R. W. [Nonlinear optics] $3^{\text {rd }}$ Ed.,, Academic Press, Cambridge, Massachusetts, 11-15 (2008)

[8] Kogelnik, H., and Li, T. „Laser beams and resonators” Proceedings of the IEEE, 54(10), 1312-1329 (1966)

[9] Vaziri, M. R., Comment on "Nonlinear refraction measurements of materials using the moiré deflectometry". Opt. Commun. 357, 200-201 (2015)

[10] Yariv, A., [Quantum Electronics] $3^{\text {rd }}$ Ed., Wiley, Hoboken, New Jersey, 106ff (1989)

[11] Yariv, A., [Quantum Electronics] $3^{\text {rd }}$ Ed., Wiley, Hoboken, New Jersey, 121-123 (1989)

[12] Powers, P. E. and Haus, J. W. [Fundamentals of nonlinear optics], CRC Press, Boca Raton, 206-212 (2011)

[13] Sheik-Bahae, M. and Said, A. A., Hagan and D. J., Soileau and M. J., \& Van Stryland, E. W. "Nonlinear refraction and optical limiting" Opt. Eng. 30(8), 1228-1236. (1991)

[14] Platt, B. C. and Shack, R. "History and principles of Shack-Hartmann wavefront sensing" J. Refract.Surg. 17(5), 573-577 (2001)

[15] Malacara, D., Servín, M. and Malacara, Z. [Interferogram analysis for optical testing] $2^{\text {nd }}$ Ed., CRC Press, Boca Raton (2011)

[16] Adair, R., Chase, L. L. and Payne, S. A. "Nonlinear refractive index of optical crystals." Phys. Rev. B, 39.5 3337 (1989) 
[17] DeSalvo, R., Said, A. A., Hagan, D. J., Van Stryland, E. W. and Sheik-Bahae, M. "Infrared to ultraviolet measurements of two-photon absorption and $\mathrm{n}_{2}$ in wide bandgap solids" IEEE. J. Quantum Elect. 32(8), 1324$1333(1996)^{\prime}$

[18] Major, A., Yoshino, F., Nikolakakos, I., Aitchison, J. S. and Smith, P. W. "Dispersion of the nonlinear refractive index in sapphire" Opt. Lett. 29(6), 602-604 (2004)

[19] Milam, D. "Review and assessment of measured values of the nonlinear refractive-index coefficient of fused silica" Appl. Optics 37(3), 546-550 (1998)

[20] Samuel, P., Ensley, T. R., Hu, H., Hagan, D. J., Van Stryland, E. W. and Gaume, R. "Nonlinear refractive index measurement on pure and Nd doped YAG ceramic by dual arm Z-scan technique" AIP Conf. Proc. 1665, 1, $060010(2015)$

[21] Aitchison, J. S., Silberberg, Y., Weiner, A. M., Leaird, D. E., Oliver, M. K., Jackel, J. L., Vogel, E.M. and Smith, P. W. E. "Spatial optical solitons in planar glass waveguides" J. Opt. Soc. A. B 8(6), 1290-1297 (1991)

[22] Lappschies, M., Görtz, B. and Ristau, D. "Application of optical broad band monitoring to quasi-rugate filters by ion beam sputtering." Appl. Optics, 45(7), 1502-1506 (2006)

[23] Dimitrov, V. and Sakka, S. "Linear and nonlinear optical properties of simple oxides. II." J. Appl. Phys. 79(3), 1741-1745 (1996) 